\title{
Norois
}

Environnement, aménagement, société

193 | 2004/4

Varia

\section{Le pays sage de George Sand}

The wise country of George Sand

\section{Max Bédéneau}

\section{OpenEdition}

\section{Journals}

Édition électronique

URL : http://journals.openedition.org/norois/705

DOI : $10.4000 /$ norois.705

ISBN : 978-2-7535-1541-3

ISSN : $1760-8546$

\section{Éditeur}

Presses universitaires de Rennes

\section{Édition imprimée}

Date de publication : 1 décembre 2004

Pagination : 7-16

ISBN : 978-2-7535-0081-5

ISSN : 0029-182X

Référence électronique

Max Bédéneau, «Le pays sage de George Sand », Norois [En ligne], 193 | 2004/4, mis en ligne le 14 août 2008, consulté le 02 mai 2019. URL : http://journals.openedition.org/norois/705 ; DOI : 10.4000/ norois. 705

Ce document a été généré automatiquement le 2 mai 2019.

(c) Tous droits réservés 


\section{Le pays sage de George Sand}

The wise country of George Sand

\section{Max Bédéneau}

\section{NOTE DE L'ÉDITEUR}

Cet article a été reçu le 27 juin 2002 et définitivement accepté le 23 août 2004.

\section{Introduction}

Dans la région Centre, nous avons installé des parcelles expérimentales dans une forêt proche de Châteauroux (fig. 1). 
Figure 1 : Localisation de l'étude

Localization of the study

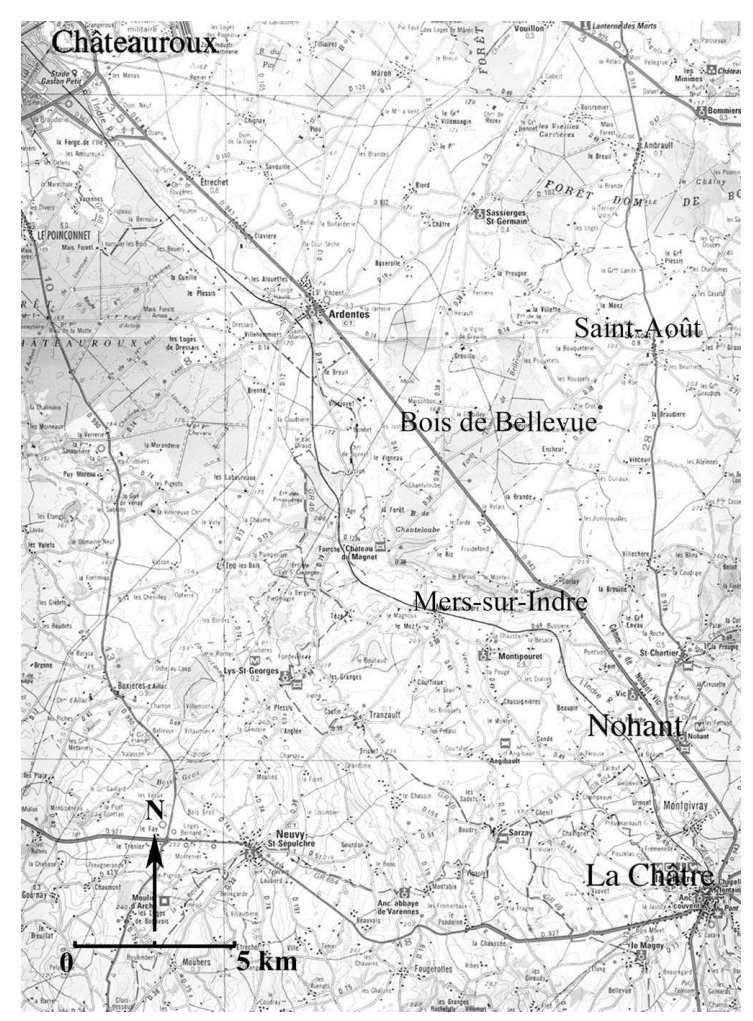

2 Ces expérimentations ont pour but de tester des techniques simples et peu onéreuses, pouvant être utilisées dans les forêts mélangées médiocres de cette région, pour en accroître la valeur des produits ligneux. En effet dans ces forêts, les interventions sylvicoles n'ont plus lieu du fait de sévères contraintes économiques, qui n'attribuent pratiquement plus aucune valeur aux produits résultant de l'exploitation. Pour chacune de ces parcelles expérimentales, il était nécessaire de connaître l'âge des arbres afin d'estimer leur potentialité de production. Dans la forêt de Bellevue, près de Nohant, nous sommes dans la campagne où George Sand situe, vers le milieu du XIX ${ }^{\mathrm{e}}$ siècle, ses romans champêtres. Or, pour cette forêt d'acquisition relativement récente par l'administration des domaines (1950), on ne possède pas d'archives. Pour obtenir des renseignements sur la succession des peuplements, nous avons recherché l'origine des propriétés dans les documents cadastraux. Il nous a semblé intéressant de faire le rapprochement entre les paysages romanesques et réels. Au travers de cette confrontation, c'est l'idée de paysage romanesque, puis son appropriation par les populations locales que nous analysons.

La région étudiée se situe entre Châteauroux et La Châtre ; elle appartient à la région forestière naturelle du Boischaut Sud. Son substrat est formé par un ensemble argilocalcaire, souvent complexe, qui correspond aux affleurements des argiles, marnes et calcaires des étages $\mathrm{du}$ Lias; il est recouvert plus ou moins régulièrement par des matériaux détritiques (Éocène ou Pliocène) et des limons éoliens (Moulin et Pelissier, 1996). 


\section{Les paysages décrits par George Sand}

\section{L'espace rural cadastré}

4 Entre 1845 et 1853 George Sand écrit cinq romans champêtres : Le Meunier d'Angibault (1845), La Mare au Diable (1846), La Petite Fadette (1849) François le Champi (1850) et Les Maîtres sonneurs (1853). La société qu'elle y décrit est présentée comme idéale, ayant échappé à la perversion des valeurs. En décrivant un monde menacé, George Sand veut en montrer la noblesse et la grandeur. Ce sont des écrits d'une femme mure et blessée, profondément engagée, obligée de se réfugier à Nohant. Elle s'y ressource, même si elle est contrainte d'écrire pour nourrir les siens. Il faut souligner que George Sand connaît parfaitement les lieux, pour y avoir grandi puis séjourné : il y a un éternel retour à Nohant qui rythme la vie de l'auteur (Barry, 1982).

5 Nous avons reconstitué l'occupation des sols en trois endroits décrits ou traversés par George Sand: le bois de Chanteloube où se déroule une partie de l'action de La Mare au Diable, le bois de Bellevue, ancienne lande où est située l'expérimentation présentée en introduction, et les bords de l'Indre sur la commune de Nohant-Vic (fig. 1).

6 Nous avons utilisé les plans et la matrice cadastrale établis par les services des impôts vers 1840 (plan dit Napoléon) et en 1988. La superposition des plans ainsi obtenus sur la photographie aérienne IGN de1988 nous a permis d'appréhender les changements survenus dans l'occupation des sols.

7 Les plans et matrices cadastraux sont d'une grande exactitude. Ils sont constamment remis à jour par les services des Impôts, en concertation avec les maires des communes. Certains détails peuvent être supprimés du fait des changements de classement des terres. Par ailleurs, les limites des communes peuvent varier dans le temps. Il peut également y avoir des erreurs (volontaires ou fortuites) dans le classement des parcelles cadastrales. Pour les communes qui nous intéressent, les cadastres de 1832, 1841 et 1842 sont les premiers jamais établis ; on peut supposer qu'ils contiennent peu d'erreurs. En 1988, nous avons pu vérifier la bonne affectation des parcelles cadastrales sur la photographie aérienne. La superposition des plans sur la photographie aérienne provoque des distorsions, qui modifient plus ou moins les contours des entités repérées (champs, prés, etc.); ce défaut est minimisé du fait de la petitesse des zones étudiées. Comme on ne s'intéresse pas aux pentes et surfaces, on n'a pas effectué de corrections.

8 Les plans de Mers (1832 et 1988), Saint-Août (1842 et 1988) et Nohant-Vic (1841 et 1988) sont présentés sur les figures 2, 3 et 4 . Ils couvrent chacun une surface d'environ 120 à 230 hectares. 
Figure 2 : Occupation des sols sur la commune de Mers-sur-Indre (bois de Chanteloube) Land use on the town of Mers-sur-Indre (wood of Chanteloupe)

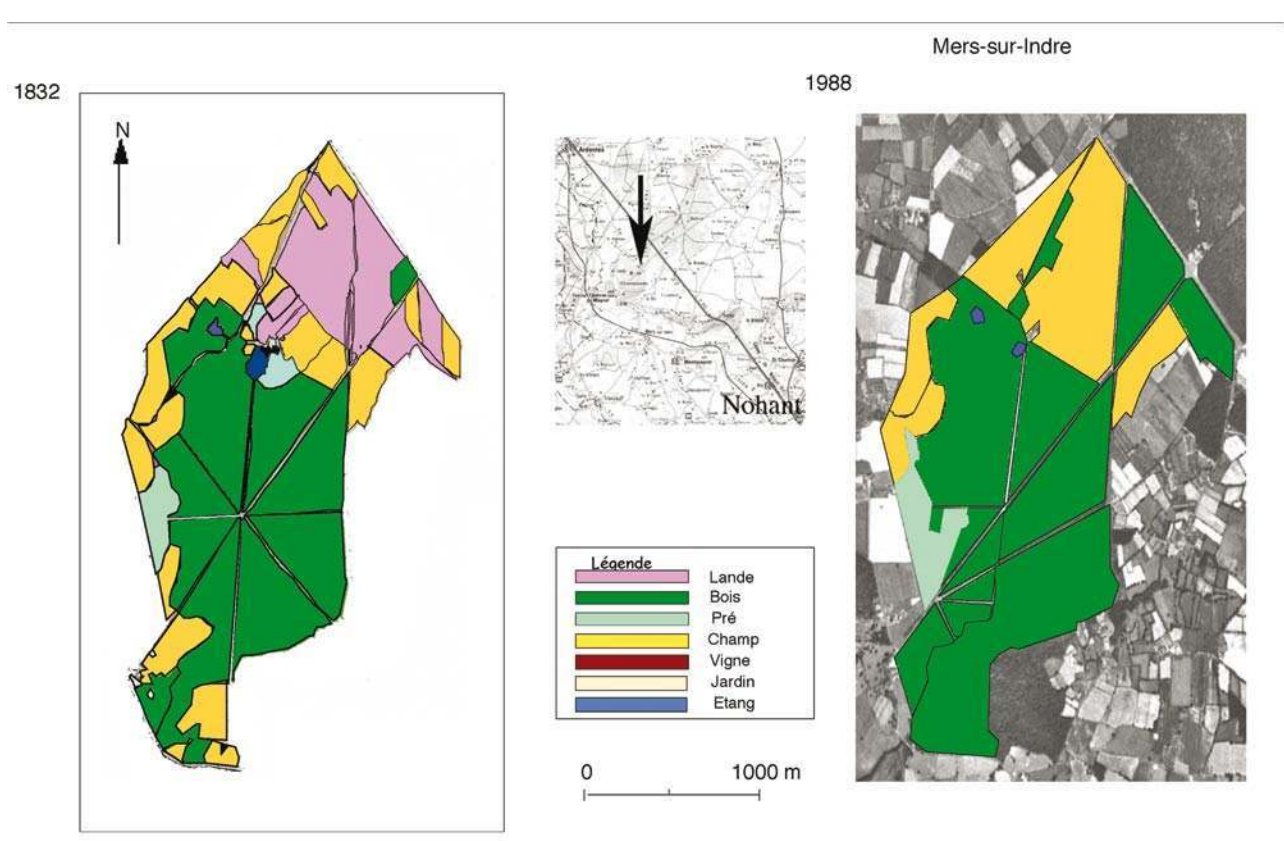

Figure 3 : Occupation des sols sur la commune de Saint-Août (bois de Bellevue) Land use on the town of Saint-Août (wood of Bellevue)
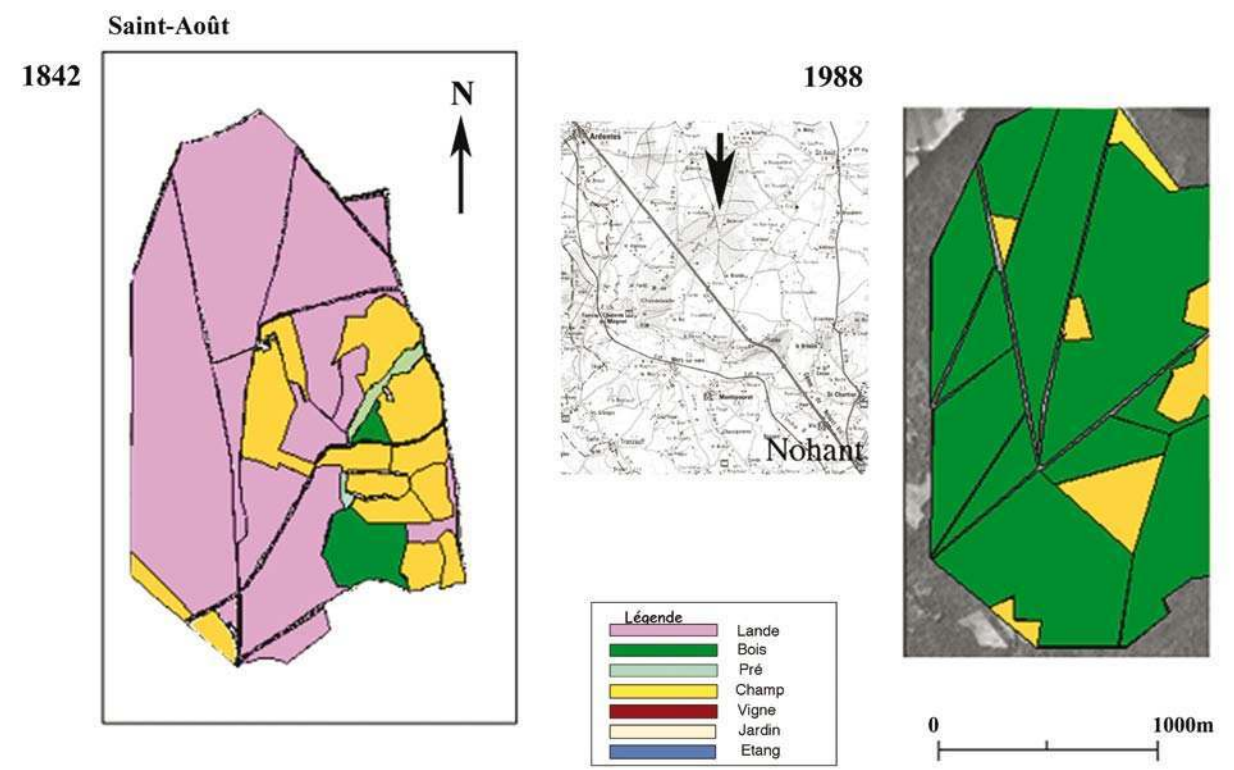
Figure 4 : Occupation des sols sur la commune de Nohant-Vic (bords de l'Indre) Land use on the town of Nohant-Vic (edges of Indre river)

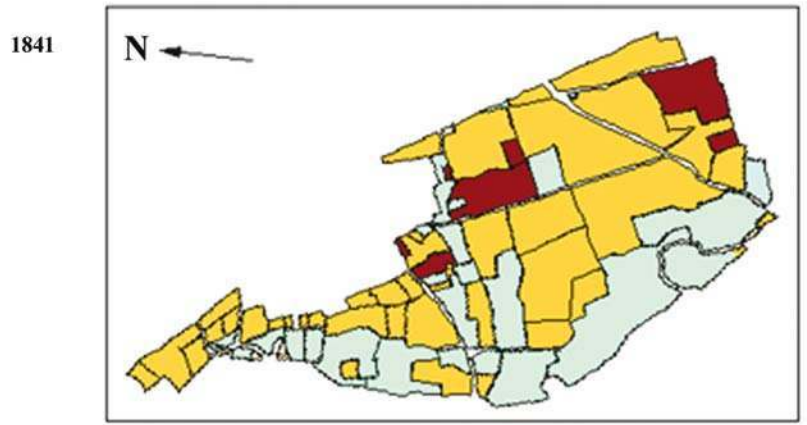

1988

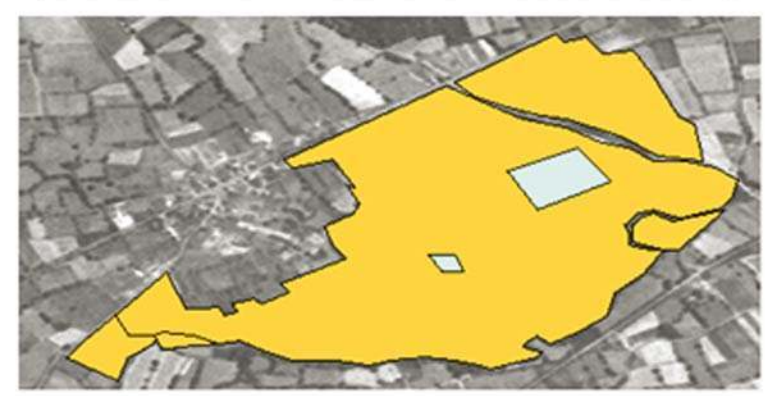

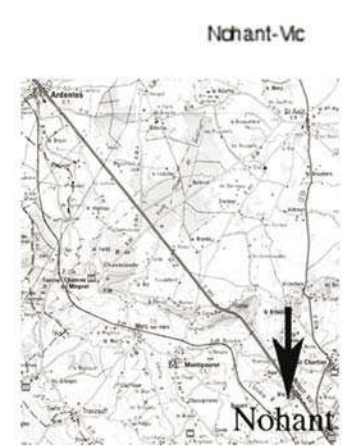

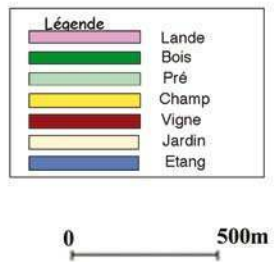

Nos trois sites révèlent trois situations différentes :

- dans le bois de Chanteloube, la forêt et les terres agricoles présentent à peu près les mêmes délimitations, quelques substitutions d'usage ont eu lieu entre forêt, landes et terres agricoles (fig. 2),

- dans le bois de Bellevue, la lande et les terres ont été boisées, seuls subsistent quelques champs (fig. 3),

- sur les bords de l'Indre, les champs et les prés existent encore aujourd'hui avec les mêmes limites, il n'y a plus de vignes, les prés ont disparu au profit de champs, il y subsiste de nombreuses haies. (fig. 4).

À Mers-sur-Indre et à Saint-Août, du temps de George Sand, le Comte Corton de Mabrillat, demeurant aux Mignaux (sur la commune de Mers), possède, à lui seul près de $85 \%$ de la surface agricole et forestière. À Nohant-Vic, George Sand est la principale propriétaire avec 224 hectares (dans le secteur retenu, elle possède 9 hectares, sur un total de 121). Le second gros propriétaire de la commune est le Comte de Charral, résidant à Voiron, en Isère (94 hectares). Neuf propriétaires possèdent $39 \%$ de la surface de la commune; parmi eux, on reconnaît certains de ceux décrits par George Sand: des nobles, des affairistes de Châteauroux ou de La Châtre, des veuves aisées. Les terrains non possédés par ces notables sont de petite surface; leurs propriétaires résident sur la commune et exercent les métiers de journalier, tisserand, meunier, aubergiste, sabotier, cultivateur.

D'après le cadastre, le paysage d'alors est celui d'une campagne avec des haies, bordant de petites pièces de terre labourable et de prés, parsemée ici et là de quelques bois (le Bois de Chanteloube ne fait que 188 hectares) et landes. Le terrain nommé aujourd'hui Bois de Bellevue appartenait au Comte Corton de Mabrillat, mais ce n'étaient que de grandes landes qui ne devinrent bois qu'en 1875, lorsque furent plantés les premiers chênes. Sur Nohant-Vic, entre la route de Châteauroux à La Châtre et l'Indre, un lacis de très petites parcelles de vignes (quelques centaines de mètres carrés chacune) appartenaient 
exclusivement à des propriétaires locaux. Les landes, bois, prés et champs occupent des places distinctes : les pâturages le long de l'Indre, puis plus haut sur le versant les terres labourables, et enfin les landes et bois plus au nord sur le plateau. Les haies sont soigneusement entretenues, car elles produisent de la litière et de la nourriture pour le bétail. En effet, l'agriculture d'alors (1830-1880) dépend de la fertilisation des terres par le fumier, et donc du nombre d'animaux produisant ce fumier (Morlon, 1998). La première révolution agricole commence à peine en France lorsque sont écrits les romans champêtres.

La parcelle où se situe notre expérimentation a été vendue, comme le reste du domaine, par le Comte Corton en 1875. C'est à cette époque que les premiers chênes furent plantés ; des châtaigniers furent introduits en sous-étage un peu plus tard, vraisemblablement dans les années 1890. À partir du début du siècle, au cours des ventes successives, la propriété est morcelée en petites parcelles; le Bois de Bellevue est acquis par l'administration des Domaines vers 1950 et devient forêt domaniale.

\section{La nature revisitée}

Les actions des romans de George Sand se situent dans un espace très restreint, un cercle de 10 kilomètres de diamètre environ autour de Nohant (fig. 5). Ceci rend aisée la perception des lieux sur le terrain (bord de rivière, bois, mare, etc.). C'est, en revanche, terriblement trompeur car les éléments du paysage sont décalés.

Figure 5 : Les lieux des romans champêtres de George Sand Localization of George Sand's countryside novels

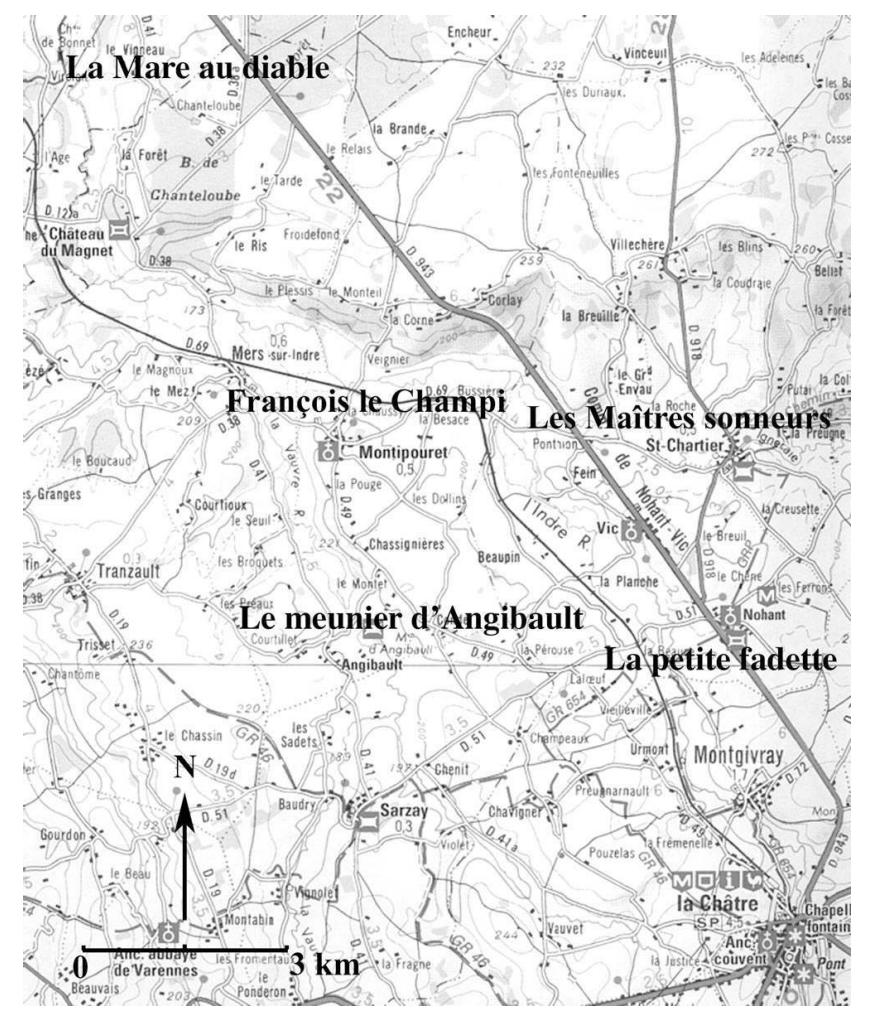

Pour des raisons diverses, la première étant de protéger sa famille, George Sand masque les lieux, les débaptise, les déplace. Cette démarche a été analysée par M. Caors (1989, 
1993), qui a montré que ces paysages, tout en étant modifiés, étaient empreints d'une certaine réalité. Elle a démonté la mécanique utilisée par Sand pour modifier ces paysages et en isoler les constituants. Les acteurs de ses romans sont des paysans locaux, souvent en lutte avec les propriétaires terriens, proches de ceux que George Sand rencontrait régulièrement à Nohant. Très influencée par J.-J. Rousseau elle enjolive les travaux agricoles et sublime les hommes qui travaillent aux champs : «Le travail est salutaire à tous, il entretient la joie et distrait de la peine, ce qui veut peut-être dire qu'il est fait pour tous, dans les idées du bon Dieu» (Le Meunier d'Angibault, 1845). À la fois par tempérament et par principe, elle embellit la réalité et idéalise les personnages : "L'art n'est pas une étude de la réalité positive ; c'est une recherche de la vérité idéale » (La Mare au Diable, 1846). Défendant les droits du cœur contre Flaubert, elle s'attache plus à décrire les sentiments ressentis par ses personnages que l'exacte réalité dans laquelle ils se meuvent. C'est pourquoi les paysages ne sont pas amplement décrits, juste un bref aperçu d'un champ, d'un chemin, d'une habitation, qui pourraient se situer n'importe où en France. Mais l'art de George Sand est de recomposer divers éléments du paysage qui l'entoure pour faire croire à l'existence de fiction paysagère. Elle fait de la nature berrichonne un décor paysager, et l'auteur nous « transmet la nature comme il la voit, et non plus comme il la voudrait peindre, l'intériorise à ses mouvements, à ses réactions, à sa pensée même. » (Caors, 1989).

George Sand tait son statut de propriétaire, omet ses riches voisins et ne parle pas non plus du développement naissant des techniques agronomiques, qui rendraient moins rude le travail des agriculteurs. En revanche, elle ne manque pas de vilipender les paysans parvenus, qui n'utilisent pas les moyens les plus élégants pour agrandir leurs propriétés. Et ce d'autant plus qu'elle connaît de graves problèmes financiers: elle vit avec les apparences de la richesse, sans la fortune correspondante; ne se plaint-t-elle pas que Nohant ne rapporte que 4000 francs par an alors que Chopin en un concert en gagne 6000 (Chalon, 1991).

16 Comme éléments de cette nature, elle retient d'abord l'eau, tant les ruisseaux multiples qui abondent dans la vallée de l'Indre que les mares et marais au milieu des bois et des landes. Elle retient aussi les haies, omni présentes dans le décor et quelques arbres épars (peupliers, trembles, aunes, chênes, ormes, bouleaux, hêtres). Enfin elle cite la terre (les champs) soumise par la volonté humaine et en retour nourrissant ceux qui la soignent. Elle va jusqu'à inventer un nom pour cette partie de la vallée de l'Indre : la Vallée Noire ; elle invente aussi la Mare au Diable, à laquelle l'administration des Impôts attribue un lieu qui apparaît sur le plan cadastral en 1987.

\section{Persistance des paysages revisités}

\section{L'espace rural actuel}

En 1843, la superficie des terres labourables, prés, jardins, vignes et pacages boisés occupait $98 \%$ de la surface de la commune de Nohant-Vic.

Les résultats du recensement général agricole de 1988 sont présentés dans le tableau 1. 
Tableau 1 : Résultats du recensement général agricole de 1988 Results of the agricultural general census of 1988

\begin{tabular}{|c|c|c|c|}
\hline & Nombre d'habitants & Nombre de chefs d'exploitation & $\begin{array}{c}\text { Part de la superficie agricole par rapport } \\
\text { à la superficie de la commune }\end{array}$ \\
\hline Mers sur Indre & 451 & 45 & $50 \%$ \\
Nohant-Vic & 480 & 41 & $70 \%$ \\
Saint-Août & 780 & 77 & $68 \%$ \\
\hline
\end{tabular}

Le système de polyculture élevage a été remplacé par des cultures céréalières sur les parties les plus riches. Les sols humides, à potentiel agronomique limité où le bocage est encore dense, sont consacrés à l'élevage bovin. L'intensification de l'agriculture, là où elle a pu avoir lieu, a fait évoluer le paysage : les haies subsistent, entretenues différemment, et les vignes ont disparu. Du fait de la persistance des haies, le paysage a peu évolué autour de Nohant-Vic, principalement à cause des contraintes hydriques et des pentes fortes. En revanche, il s'est modifié autour de Saint-Août: les parties en brandes ont été reboisées pour donner naissance au Bois de Bellevue, plus rémunérateur par la chasse qu'on y pratique, que par le bois qu'on en tire. En effet, un rôle non négligeable de récréation est dévolu à certaines forêts de l'Indre, soit par la proximité de villes - la forêt domaniale de Châteauroux -, soit par le tourisme vert, ou la chasse. Elles sont aménagées en conséquence par les gestionnaires forestiers: larges allées, grands espaces de stationnement, réhabilitation d'anciennes maisons forestières en rendez vous de chasse. Une composante importante du paysage actuel est l'apparition de maisons nouvelles où vit la population travaillant dans les villes ou dans les zones d'activités proches (Ardentes, Châteauroux, La Châtre). Par rapport aux paysages de 1843, quelques éléments occupent la même place : un étang et une ferme dans le bois de Chanteloube, des haies le long de l'Indre, fermes et terres à l'orée du bois de Bellevue. Cependant, les arbres isolés, le bocage, les vallons, les routes étroites, la rivière, forment un paysage qui est proche de ce qu'a décrit George Sand, au moins dans la vallée de l'Indre. Le réseau de haies est visible sur la figure 6, issue de la photographie aérienne de 1988 de Nohant-Vic. 
Figure 6 : Les haies le long de l'Indre Hedges along Indre river

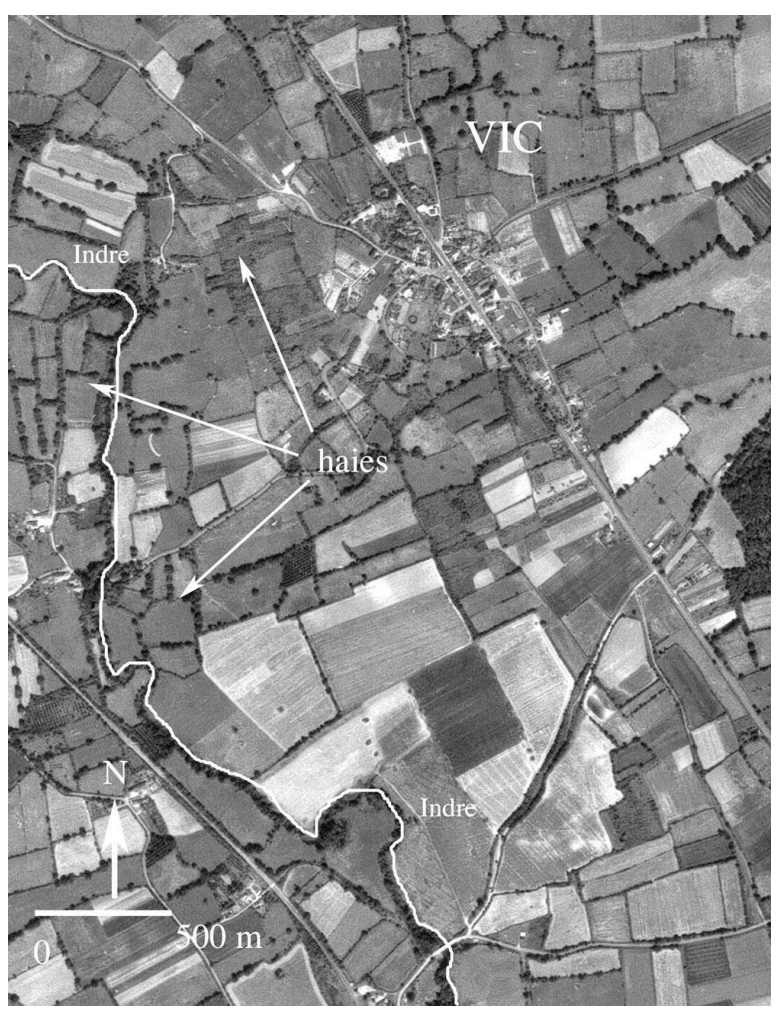

(photographie aérienne IGN : 23 juin 1988 - FD 36 - 1/30 000 - n²97)

\section{La présence de George Sand}

Dans cette campagne, on ne peut que remarquer le paysage, tant sont présents les aires de contemplation ou les circuits fléchés. Le promeneur est invité à découvrir, le long de sentiers initiatiques, les décors des romans champêtres, tout comme il y a 150 ans, un pays sage, paisible, reposant. Dès que l'on arrive près de Nohant, on est frappés par la présence de nombreux panneaux informatifs et publicitaires faisant référence à l'œuvre de George Sand. Auberges, restaurants, hôtels, allées forestières, circuits touristiques pédestres, cyclistes ou automobiles, tout est prétexte à récupérer son nom et ceux de ses romans et de ses personnages : la demeure du médecin de George Sand, à Saint-Chartier, est devenu un luxueux hôtel dominant la Vallée noire, curieusement nommé La Vallée Bleue; l'auberge face à l'église de Nohant porte le nom de La Petite Fadette. Des rues, des avenues, des squares portent le nom du grand auteur local : à La Châtre, le lycée GeorgeSand est situé Avenue George-Sand, alors que le collège George-Sand est, lui, impasse George-Sand. Les chambres d'hôtes et les gîtes ruraux se développent le long de ces circuits. Les manifestations culturelles sont multiples: les «Fêtes romantiques de Nohant » (fin juin), les « Rencontres internationales de luthiers et maîtres sonneurs » à Saint-Chartier (mi-juillet), les Rencontres internationales Frédéric Chopin à La Châtre (fin Juillet). Les manifestations de Nohant et de La Châtre sont organisées par l'Association Musique au Pays de George Sand, qui a pour présidents d'honneur des personnalités célèbres, les « Rencontres de Saint-Chartier » sont préparées par le Comité George Sand de La Châtre. Ces manifestations bénéficient du soutien du ministère de la Culture, de la 
Région Centre, du département, et d'entreprises privées d'ampleur internationale; les radios locales couvrent l'événement. Les "Rencontres de Saint Chartier", créées à l'occasion de l'anniversaire du centenaire de la mort de George Sand en 1976, accueillent près de 30000 personnes. Dans Les Maîtres sonneurs, George Sand place à Saint-Chartier les joutes musicales, et invente un groupe de sonneurs, « Les Gars du Berry » : aujourd'hui, ce groupe, né de l'imagination de notre auteur, existe réellement. Les deux autres manifestations regroupent environ 8000 personnes. Ces grandes manifestations se tiennent dans des lieux préservés, peu exploités.

21 Plus récemment s'est ouvert, avec les mêmes soutiens, le Centre international GeorgeSand et le Romantisme (CIGSR) au château d'Ars à Lourouer-Saint-Laurent. Il existe donc un tissu très dense de personnes qui perpétuent le souvenir de la «bonne dame» de Nohant, afin notamment de l'utiliser pour le développement touristique en participant ainsi de manière notable à l'économie locale. La gestion de l'espace par les agriculteurs détruit certains éléments des paysages de George Sand (par exemple, l'agrandissement des pièces de terres ouvre de plus larges perspectives).

\section{Conclusion}

Pour mener cette étude, on a fait l'hypothèse que l'occupation des sols était comparable entre le milieu du XIX ${ }^{e}$ siècle et la fin du $\mathrm{xx}^{\mathrm{e}}$ siècle. Pour cela, on s'est appuyé sur les documents du cadastre, à partir desquels est fixée l'assiette des différents impôts fonciers. Cependant, il ne faut pas perdre de vue que les pratiques agricoles et forestières de 1850 sont bien différentes de celles d'aujourd'hui, non seulement par les techniques, mais aussi par la diversité des cultures. Plus que le paysage proprement dit, on peut reconstituer une occupation des sols, en faire une étude diachronique, et la relier aux romans champêtres.

Il apparait comme réel que George Sand a modifié les paysages, et la réalité qui l'entouraient. Il apparaît aussi comme établi que son œuvre a encore une audience et que chaque année de nouveaux romantiques se rendent à Nohant. On est donc bien en face d'une "manipulation de paysage " (Larrère, 1994). Le décor romanesque n'est plus un pays tenu par de grands propriétaires, dominé à coups de procès et de misère, c'est un lieu charmant où sont entretenus les haies, les prés, les champs, les chemins et les routes sans heurts et sans pleurs. Cette démarche n'a d'ailleurs rien de choquant: l'auteur littéraire est un idéaliste qui ramène tout à son sujet, le regard porté sur la nature n'est pas objectif. Il faut préciser que, où qu'il soit apparu, le romantisme se caractérise par le libre cours donné à l'imagination et à la sensibilité individuelles, qui le plus souvent traduisent un désir d'évasion et de rêve. À travers les constantes du romantisme européen (réveil de la poésie lyrique, rupture avec les règles et les modèles, retour à la nature, recherche de la beauté dans ses aspects originaux et particuliers), chaque nation a laissé éclater son génie propre.

Mais George Sand a si bien su décrire son décor quotidien que ses lecteurs se le sont approprié. C'est la construction même des romans champêtres - décors de faible ampleur, temps ralenti par des personnages se déplaçant au rythme des travaux agricoles - qui donne la sensation que le paysage est inchangé. Et, bien que les affectations des parcelles et certaines cultures aient changé, des éléments forts du paysage de 1835 subsistent 
autour de Nohant. Mais il est vrai que le bois de Bellevue n'est plus une plaine recouverte de bruyères, et que le bois de Chanteloube est plus étendu.

On peut se poser la question de la coexistence des pratiques agricoles - qui à terme peuvent altérer le décor de George Sand - et des activités touristiques et culturelles qui, en revanche, demandent des invariants paysagers.

Les espaces demandés aujourd'hui par le public relèvent de cette démarche, à la fois quelque chose de rationnel, sécurisant, et un domaine de rêve et d'irrationnel, finalement propre à chaque région. On retrouve cet ensemble à Nohant en Berry, peut être plus qu'au Grand Bé de Saint-Malo, qu'à Ermenonville ou à Villequier, tous autres lieux hautement inspirés du Romantisme. C'est dans doute parce que George Sand, dans la mise en scène de ce pays sage, a fait évoluer des gens que l'on croirait côtoyer aujourd'hui. Cette étude pourrait être poursuivie en partageant plus intimement le quotidien des gens qui travaillent dans la Vallée Noire et entretiennent les paysages de George Sand.

\section{BIBLIOGRAPHIE}

BARRY (J.), 1982. - George Sand ou le scandale de la liberté, Paris, Seuil, 568 p.

CAORS (M.), 1989. - Le Berry de George Sand Géographie Imaginaire, M. Caors (éd.), 433 p.

-, 1993. - George Sand de voyages en romans, Royer, 271 p.

ChaLON (J.), 1991. - Chère George Sand, Paris, Flammarion, 475 p.

LARRÈRE (R.), 1994. - « L'art de produire la Nature, une leçon de Rousseau », Courrier de

l'Environnement de l'INRA, (22), p. 5-13.

MORLON (P.), 1998. - « Vieilles lunes ? Les normes pour les bâtiments d'élevage ont cent cinquante ans, le code de bonnes pratiques agricoles en a cent... ", Courrier de l'Environnement de l'INRA, (33), p. $45-60$.

Moulin (J.), Pelissier (P.), 1996. - Notice explicative de la Carte des sols de Ardentes, chambre d'agriculture de l'Indre, $293 \mathrm{p}$.

SAND (G.), 1992. - La Mare au Diable, Paris, J'ai Lu.

-, [1990]. - Le Meunier d'Angibault, Paris, Glénat.

-, [1976]. - François le Champi, Paris, Le Livre de Poche.

-, [1973]. - La Petite Fadette, Paris, Le Livre de Poche.

-, [1994]. - Les Maîtres sonneurs, Paris, Glénat.

\section{Sources}

\section{Archives Départementales de l'Indre :}

Matrice Cadastrale de Mers-sur-Indre. 
Matrice Cadastrale de Saint-Août.

Matrice Cadastrale de Nohant-Vic.

$62 \mathrm{~J}$ : Les Familles, les Ventes de terres, Acquisition des terres de Nohant.

\section{Centre Départemental des Impôts Fonciers :}

Plan cadastral de Mers-sur-Indre, feuille N1, 1832, 1987.

Plan cadastral de Nohant-Vic, feuille D2, 1841, 1988.

Plan cadastral de Saint-Août, feuille N1, 1842, 1987.

\section{Photographies aériennes IGN :}

1988 : Photographie 297, 299, 301, 356, 358, 360, 362, 367, 371, 373, 375.

\section{RÉSUMÉS}

Dans le cadre d'études de productivité de forêts mélangées de la région Centre, nous avons été amené à rechercher dans les archives l'origine d'une forêt située prés de Nohant (Indre). Nous avons suivi l'évolution de l'occupation des sols au cours des 150 dernières années. La zone étudiée couvre partiellement celle qui a été décrite par George Sand dans ses romans champêtres. Par rapport aux paysages de l'époque, il apparaît que l'écrivain, pour diverses raisons, a largement modifié, sans les altérer, les paysages d'alors, embellissant une réalité qui ne méritait pas toujours de l'être. Elle a su créer un pays sage, havre de paix et de repos. À travers l'évolution de trois grands types de paysages de la Vallée Noire (forêt, bocage, champ), nous décrivons l'utilisation de l'espace, sa transformation, et son aspect actuel physique et humain. La récupération du nom de «George Sand » permet l'exploitation, dans ce milieu rural en évolution, des paysages inventés, presque vrais, tant par des acteurs locaux que régionaux.

Within the study framework on the productivity of mixed forests in the Central Region of France, we investigated the archives to find out the origin of a forest located near Nohant (Indre).

We followed the succession of the occupation of the land during the last 150 years. The studied zone partially covers that which is described by George Sand in her pastoral novels.

Our investigation shows that the writer modified the landscapes in her descriptions by changing the reality. It has been most likely due to her desire to create a countryside that was peaceful and well managed.

Throughout the evolution of the three great types of landscapes of the Vallée Noire (forest, grove, field), we describe the use of space, its transformation, and its current physical and human aspects.

The recent use of the name "George Sand" allows local and regional planners to add value to this rural and changing landscape.

\section{INDEX}

Mots-clés : cartographie, documents d'archives, George Sand, paysage, occupation du sol Keywords : cartography, documents of files, George Sand, land use, landscape 
AUTEUR

MAX BÉDÉNEAU

Unité Amélioration, Génétique et Physiologie forestières - INRA, max.bedeneau@orleans.inra.fr 